\title{
Multidimensional Adaptations for Open Learning Management Systems
}

\author{
Silvia Baldiris ${ }^{1}$, Olga C. Santos ${ }^{2}$, David Huerva1, Ramón Fabregat ${ }^{1}$ and Jesus G. Boticario² \\ Institute of Informatics and Aplications (IIiA), Universidad de Girona, Spain \\ \{baldiris,dhuerva\}@eia.udg.es,ramon.fabregat@udg.es \\ ${ }^{2} a D e N u$ Research Group, Artificial Intelligence Department, Computer Science School, UNED \\ \{ocsantos,jgb\}@dia.uned.es
}

\begin{abstract}
Our work is focused on alleviating the workload for designers of adaptive courses on the complexity task of authoring adaptive learning designs adjusted to specific user characteristics and the user context. We propose an adaptation platform that consists in a set of intelligent agents where each agent carries out an independent adaptation task. The agents apply machine learning techniques to support the user modelling for the adaptation process.
\end{abstract}

\section{Motivation}

Information and communication technologies have been adopted in the learning process via the design and development of learning management systems (LMS) with the aim to remove some of the barriers in traditional face to face settings (e.g. geographic and temporal) and to add potential advantages, such as the possibility of better addressing the individual user's needs in a personalized and inclusive way. However, using LMS for instruction in order to support the learning activities of students requires an extra effort from the teacher.

Moreover, there is a strong interest in supporting lifelong learning processes, in which learning is integrated throughout people's lifetime due to the rules of the current knowledge-based society, which forces all citizens to daily improve a wide variety of competences to succeed at the workplace. In this context, the efficiency of the learning process is measured according to the users' achievement of their competences. Competences are complex processes that people put into play in order to solve problems and to carry out activities. Users and their characteristics are key elements in a competence-based learning process development, especially considering that the very evolution over time of those characteristics reflect the expected acquisition of users' competences.

Our work is focused on a technological support for generating learning designs adjusted to the user characteristics and the user context.
The approach is based on an adaptation platform called ADA + that consists in a set of intelligent agents where each agent carries out an independent adaptation task. Machine learning techniques are applied for the user modelling for adaptation process. This platform has been integrated in dotLRN LMS.

\section{Conceptual Design}

When the adaptation idea is introduced in the context of a virtual learning process, the starting point is the modelling of the teaching-learning process as in the traditional (face to face) process. In this section, we begin with a general description of the elements included in this process, continuing with the two main tasks that are the base of our proposal for the personalization process to be done in a LMS: the user modelling task and the adaptation task.

The first one covers three main objectives: 1) define the user characteristics that are relevant to in a specific context to generate useful adaptations, 2) define how these characteristics can be obtained or inferred, and 3) define how these characteristics contribute to the adaptation process. The second one describes the adaptation mechanism itself, that is, how the users' characteristics are managed to generate an adjusted learning design path.

Our interest in the ADAPTAPlan project [2] is to combine different types of adaptation in order to cover more completely the heterogeneity of users [23]. This complete adaptation consist in 1) delivering the learning objects adjusted to the specific competence level of the users 2) delivering ranked learning objects according to the user learning style; 3) suggesting future actions to users according to their collaborative behaviour; 4) guaranteeing access to the learning objects according to the device characteristics.

\subsection{Teaching Learning Process Description}

Our approach requires the modelling of teachinglearning process based on the following elements:

1) Competence model permits to define appropriate 
performances that should be demonstrated by a person on a specific context. Competences are described using IMS - RDCEO specification [3].

2) Course Structure Model consists in the course structure that supports students' achievement of the proposed competences. This structure is defined through a particular ontology for each course and it is stored in a Domain repository.

3) Learning Resources and Metadata Model have been modelled as atomic units related to topics in the course structure and specifics competences through IMS-MD [4]. We are using Fedora Commons as learning object repository [5].

4) Recommendation Model describes what should be recommended in the context of a virtual learning process [6].

5) Evaluation Model consists in different assessment elements, theoretical and practical, related to evidences attribute in the competence definition $[17,18]$.

\subsection{User Modelling}

The user modelling task defines and maintains upto-date user models [1]. Brusilovsky defines basically two types of user models, feature-based models and stereotype models. The first one models changeable features of users, whereas the second defines groups of users that share specifics characteristics.

ADAPTAPlan approach combines both types of user models to generate multidimensional adaptations. In particular we are addressing three features of the learner: competences level, learning styles and access device preferences. In short, the first one is the result of overlay goals modelling and dynamic stereotypebased clustering approach; the second one is a static stereotype model and the third one is a dynamic stereotype model that depends on the user context, in particular, on the type of access device used.

We generate three partial user profiles in IMS-LIP [13] to cover the characteristics afore mentioned:

- Learning Style Profile. Learning Style is an enrich abstraction of Felder categorization and it is inferred through the application to users of the Felder's Index of Learning Style [9]. The user model is constructed as described elsewhere [2].

- Competences Profile. We consider two different types of competences, generic and specific [10]. The specific competences are directly related to a specific occupation while the generic, also called transverse, have mobility in various fields and are transferable to a multitude of functions or training programs. Collaborative competences are a special type of generic competences.
- Access Device profile. Our approach considers some characteristics about user access device [7], in particular, some hardware and software characteristics.

The model presented above is stored and maintained on an IMS-LIP service [24]. This server is accessed by the $\mathrm{ADA}+$ adaptation platform via web service communication, specifically SOAP protocol [11] when necessary. Implementation details are described in next sections.

\subsection{Adaptation Process}

The adaptation process supports different types of adaptations based on each partial profile. We have developed an adaptation platform, which is independent of any specific LMS, in order to support adaptations in a flexible and interoperable way. Our schema takes into account some considerations: 1) using an intelligent agent platform, where different autonomous processes consider aforementioned user's characteristics while collaborating in the generation of the adaptive features; 2) constructing the user model by applying machine learning techniques; 3) using web services as integration mechanisms.

Adaptation platform consist in a data structure, an intelligent agents platform and the deliver context. In [23] a detail description of it can be found.

Data structure collects the necessary data to support the user modelling and adaptation processes. In particular, the elements of the learning process mentioned before and the user data. User data come from basically two different sources, data from the user profile and data about the user's interaction on the LMS. Data structures also contain the CC/PP profiles in a Sesame repository that consists in RDF access device descriptions $[7,24]$.

$A D A+$ intelligent agents platform consists in different intelligent agents that carry out diverse tasks [8]. Some of these agents carry out adaptation tasks using machine learning techniques from the wellknown Weka suite [20] in order to support 1) the user modelling (e.g. the Collaborative Competence Adapter) and 2) the adaptation process itself (e.g. the Learning Style Adapter). Other agents carry out integration tasks such as the Yellow Pages Agent and the Communicator agent.

The Main Adapter is the principal adaptation process. It uses data provided by all the other agents and planning techniques to generate an IMS Learning Design [22] adjusted to the user characteristics. Description of this process can be found in [14]. 
The deliver context is the LMS presentation layer, where the course contents and activities are presented. In our case, we are working with dotLRN open source LMS. There, two components can benefit from the information computed in ADA+: 1) the IMS-LD player, which presents an adapted learning design to the learner and 2) the A2M Recommender System [12] that offers dynamic recommendations to the learners.

\section{Adaptation Platform ADA+}

The Adaptation platform has been modelled as an independent set of JADE intelligent agents [16] that collaborate to support the adaptation process to users as was mentioned above.

In order to publish the services offered by each of the adapter agents we have developed a system that it allows a process implementing an XML-RPC [15] client to request the agents' services. The system is composed of a Servlet implementing an XML-RPC server, a Yellow Pages agent and a Communicator Agent.

When a process wants to request the service offered by an agent it sends a request with the service name and parameters to the servlet implementing the XMLRPC server. This servlet, which acts as a gateway between the process making the request and the agent platform, sends the request to a Communicator Agent. The task of this agent is to find out which agent offers the service being requested and to forward it the request. To do this it communicates with the Yellow Pages agent, to which the agents register the service they offer. Once the agent that provides the service receives the request it processes it and sends a reply to the Communicator Agent which forwards it by means of the servlet to the process that made the request. Requests are codified as a string, which identifies the service being requested, and an array containing the request parameters, while responses are codified with an array of values. Next sections describe each agent's behaviour in adaptation platform.

\subsection{Competence Adaptation}

We consider two types of competences that have two different adaptation processes. Next, the specific and collaborative competences adapters are described.

\section{Specific Competences Adaptation}

Specific Competences Adapter carries out the adaptation according to user specific competence level. User Competence level is located is stored in the IMSLIP server [24]. The Adapter consists of three agents: Provider Agent, Requester Agent and Updater Agent. When the user initiates a session in a specific course, the Requester Agent recovers the information about the user's competence level according to the competences associated to the course at hand. The Provider Agent uses that information to fetch the adequate learning objects from the Fedora Commons Repository, based on the analysis of the learning objects metadata. Specifically, the label pointing to the learning object difficulty level is contrasted the competence level of the user. The Provider Agent delivers to the Communicator Agent a list of learning objects identifiers that can be studied for the particular user and send this information to the Main Adapter to support the final IMS-LD generation.

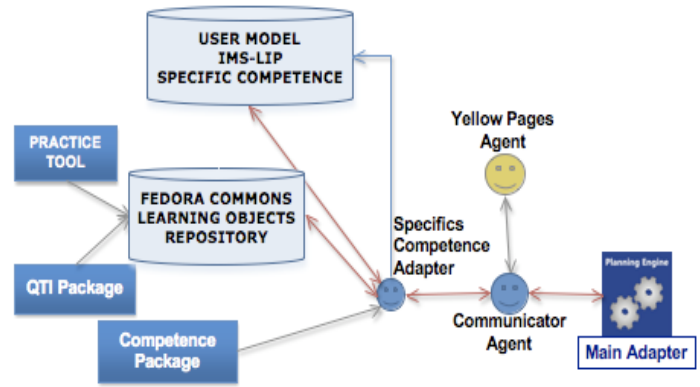

Figure 1. Specific Competences Adapter

When the user finishes a course session or a critical event happens, for instance, the student performs a test, the Updater Agent updates the student model by calculating a new competence level based on data from the interaction.

\section{Collaborative Competences Adaptation}

The purpose of this process is to support recommendations delivering according to collaborative competence level of users. Definition of Collaborative Competence Level is based on user's behaviour on different collaborative tools [19]. We have considered the problem of the student's location at a given level of collaborative competence as a grouping problem. This decision was taken because it is impossible to know in advance to which class the student belongs to [20].

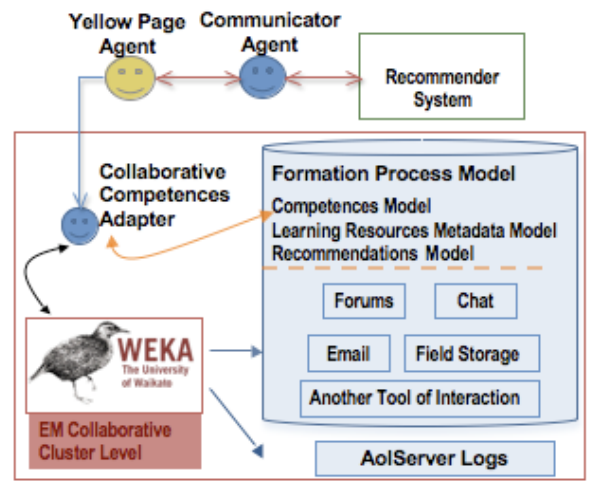

Figure 2. Collaborative Competences Adapter 
The Collaboration Model includes the construction of two sub models: 1) The Participation Model inferred through the interaction data obtained from forums, chat and other collaboration tools; and 2) The Interaction Model based on the interaction frequency of the user in a specific course.

Clustering techniques allow grouping students in subsets (clusters) of them, according to their collaboration similarities described in [19].

The Adapter is modelled using the Behaviours Design Tool of Agent Academy framework [21], which generates agents behaviours associated to machine learning algorithms, in particular, the EM algorithm. The input of grouping behaviour corresponds to a specific arff file generated by treating data from users' interactions in different collaboration tools and server logs. The Collaborative Competence Adapter locates the user in a specific collaboration level and delivers this level when requested.

\section{Learning Style Adaptation}

It has already been introduced that the Learning Style Adapter defines the best order to deliver learning objects to users and it consists of two agents, the Requester Agent and the Classifier Agent.

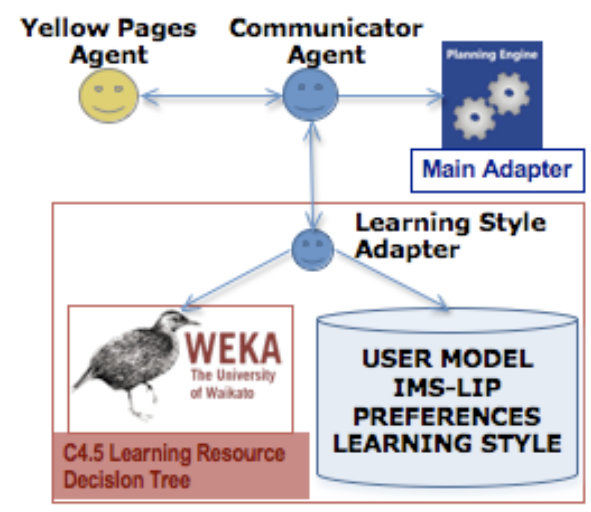

Figure 3. Learning Style Adapter

The first one recovers the information about the user learning style stored in the IMS LIP server to be used by the Classifier Agent, which provides the classification behaviour. Input of classification behaviour corresponds to a specific arff file used for J48 algorithm to generate a decision tree. The decision tree is used by the Classifier Agent to generate a specific order for each stereotype of learning style. Examples of training used by J48 algorithm come from two different sources, 1) a survey was done in order to capture teachers' opinion about preferences of each learning style with respect to each type of IMS-MD learning objects type; and 2) some students expressed their preferences about object types. Generated order is send to the Main Adapter to finally support the IMS LD generation.

\section{Context Adaptation}

The context adaptation is carried out by means of the Context Adapter agent. Context is described as a set of technological features of the user device stored in UAProf profiles and evaluated by a set of intelligent agents, living both in the user's device and the web server, which gathers context data and infer the value of a set of adaptation properties.

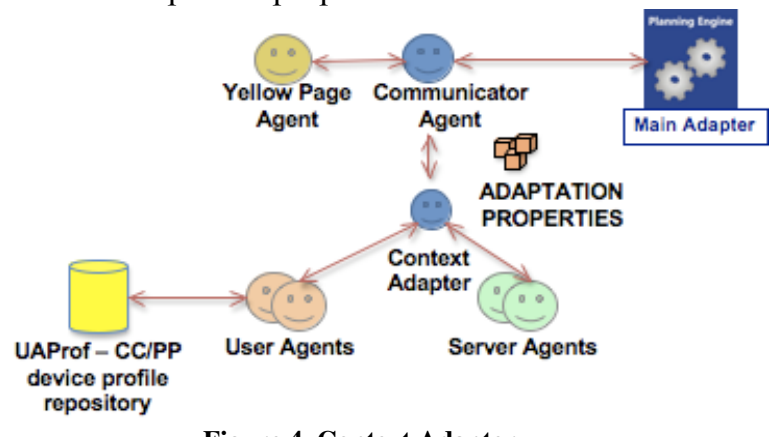

Figure 4. Context Adapter

The context adaptation in the Main Adapter is carried out in two levels, by adapting the master templates, that gives a general look to the pages of the learning environment according with the user device and offering equivalent resources at run time in the generated IMS-LD.

\section{Adaptation Results}

The adaptation results consist in the generation of IMS Learning Design [22] adjusted to user's characteristics. Each particular adapter introduced above provides the necessary data for its generation. This adaptation is developed by the Main Adapter, which includes a planning engine that generates a plan using the conditions mechanism of IMS-LD specification. This allows to develop a plan that shows or hides a given content depending on the value of the set of properties that have been considered.

User Behaviour is always tracked in the IMS-LD execution, and adapters are listening to control critical events in the learning process. Events such as assessments presentation or the session finalization modify properties in the learning design and produce a re-planning process by the Main Adapter.

\section{Conclusions and Future works}

We have presented an approach to support the learning process in an open LMS, which is based on user modelling and provides adaptive features. Our 
proposal is supported technically by means of intelligent agent technologies, machine learning techniques and web services. The adaptation process is based on three characteristics: competence level, learning style and context.

Agents from the $\mathrm{ADA}+$ adaptation platform carry out some tasks independently, creating a flexible collaborative environment among intelligent agents.

We are working on adding dynamism to user profile based in the user learning style and also for improving the collaboration profile considering the quality of the content of the user participation, and not only quantity and frequency of contributions.

\section{Acknowledgment}

Authors would like to thank the Spanish Science and Education Ministry for the financial support of ADAPTAPlan project. Thanks to the Programme Alban, the European Union Programme of High Level Scholarships for Latin America, scholarship No. E06D103680CO.

\section{References}

[1] Brusilovsky P., Millán E. User Models for Adaptive Hypermedia and Adaptive Educational Systems. (2007). The Adaptive Web, LNCS 4321, pp. 3 - 53. SpringerVerlag Berlin Heidelberg.

[2] Baldiris, S., Santos, O. C., Barrera, C., Boticario J. G., Velez, J., Fabregat, R.: Integration of educational specifications and standards to support adaptive learning scenarios in ADAPTAPlan. International Journal of Computer Science and Applications (IJCSA). Special Issue on New Trends on AI techniques for Educational Technologies. Vol 5, 1, 2008.

[3] IMS Reusable Definition of Competency. Version1.0. Final Specification. 2002.

[4] IMS Metadata 1.2.1. Final Specification. 2001.

[5] Flexible Extensible Digital Object Repository Architecture. http://www.fedora-commons.org/. 2008.

[6] Santos, O.C. and Boticario, J.G. Users' experience with a recommender system in an open source standardbased learning management system. In proceedings of USAB 2008 (in press)

[7] Merida, D., Cannataro, M., Fabregat, R. and Arteaga, C.: MAS-SHAAD a Multiagent System an Adaptive Hypermedia System. Proceedings of IJCEELL journal Special issue: Adaptivity in Web and Mobile Learning Services, 2004.

[8] Santos, O.C., Baldiris, S., Velez, J., Boticario, J.G., Fabregat, R.Dynamic Support in ADAPTAPlan: ADA+. Proceedings of CAEPIA, vol. II. 2007, p. 131-140.

[9] Felder R. M., Silverman L. K.: Learning and Teaching Styles In Engineering Education. Engr. Education, 78(7), 674-681. 1988.

[10] European Commission.: Tuning Educational Structure in Europe. Brussels. 2002.
[11] Sobernig, S. Xorb/xosoap. Remoting for OACS / .LRN. dotLRN Conference Boston, 2006.

[12] Santos, O.C., Raffenne, E, Granado, J. and Boticario, J.G. Dynamic support in OpenACS/dotLRN: Recommending actions for all. Proceedings of the Int. Conference on Community based environments, 2008.

[13] IMS Learner Information Profile. Version 1.0. Final Specification 2001.

[14] Morales, Ll., Castillo, L., Fernandez-Olivares J., and Gonzalez-Ferrer A.: Automatic Generation of User Adapted Learning Designs: An AI-Planning Proposal. 5th Int. Conf. on Adaptive Hypermedia and Adaptive Web-Based Systems. Hannover, 2008.

[15] Apache XML-RPC, http://ws.apache.org/xmlrpc/

[16] Java Agent Development Framework. JADE. http://jade.tilab.com/.

[17] IMS Question and Test Interoperability. Version 1.2.1 Final Specification, 2003.

[18] Jurado, F., Santos O. C., Redondo, M. A., Boticario, J G., Ortega M.: Providing Dynamic Instructional Adaptation in Programming Learning In proceedings of the 3rd International Workshop on Hybrid Artificial Intellingent Systems (HAIS 2008), in press.

[19] Mancera, L. Baldiris, N., Fabregat, R.: Modelling Collaborative Competence Level using Machine Learning Techniques.

[20] Data Mining with Open Source Machine Learning Software in Java. http://www.cs.waikato.ac.nz/ml/weka/.

[21] A Data Mining Framework for Training Intelligent Agents. Agent Academy. http://agentacademy.iti.gr/.

[22] IMS Learning Design. Version 1.0 Final Specification, 2003.

[23] Baldiris, S., Santos, O. C., Moreno, G., Huerva, D., Boticario J. G., Fabregat, R.: Management of learning styles, competences and access device preferences to alleviate the authoring of standard-based adaptive learning designs. 5th Int. Conf. on Adaptive Hypermedia and Adaptive Web-Based Systems. Hannover, 2008.

[24] Cuartero, A., Santos, O.C., Granado, J., Raffenne, E. and Boticario, J.G.: Mangement of standard-based User Model and Device Profile in OpenACS. Proceedings of the International Conference and Workshops on Community based environments, 2008. 